\title{
Positive Feedback, Stochasticity and Genetic
}

\section{Competence}

\author{
Rajesh Karmakar and Indrani Bose* \\ Department of Physics \\ Bose Institute \\ 93/1, Acharya Prafulla Chandra Road, Kolkata-700 009, India. \\ * Author to be contacted; e-mail: indrani@bosemain.boseinst.ac.in
}

\begin{abstract}
A single gene, regulating its own expression via a positive feedback loop, constitutes a common motif in gene regulatory networks and signalling cascades. Recent experiments on the development of competence in the bacterial population B. subtilis show that the autoregulatory genetic module by itself can give rise to two types of cellular states. The states correspond to the low and high expression states of the master regulator ComK. The high expression state is attained when the ComK protein level exceeds a threshold value leading to a full activation of the autostimulatory loop. Stochasticity in gene expression drives the transitions between the two stable states. In this paper, we explain the appearance of bimodal protein distributions in B. subtilis cell population in the framework of three possible scenarios. In two of the cases, bistability provides the basis for binary gene expression. In the third case, the system is monostable in a deterministic description and stochasticity in gene expression is solely responsible for the appearance of the two expression states.
\end{abstract}

\section{Introduction}

Positive feedback loops are common motifs in gene transcription regulatory networks and signaling cascades. The simplest such motif is the autoregulatory loop in which the proteins synthesized by a gene stimulate the production of more proteins in an autocatalytic fashion [1, 2, 3]. In most cases, the concerned gene is also expressed at a basal level, i.e., proteins are synthesized even when the positive feedback is non-functional. The autoregulatory dynamics have a nonlinear character and this combined with positive feedback may give rise to binary gene expression in a range of parameter values. The protein levels, as a result, have a bimodal distribution in a population of cells. In a fraction of cells, the protein 
level is low and in the rest of the population the level is high. Theoretical predictions of binary gene expression have been verified in experiments on single gene autocatalytic modules in bacteriophage $\lambda$ and $S$. cerevisiae [2, 4]. The experimental findings further suggest that the observed bimodality has a stochastic origin. A remarkable example of population heterogeneity, brought about by a combination of autoregulatory positive feedback and stochasticity, is provided by the bacterial population $B$. subtilis in which a fraction of the population develops genetic competence. Microorganisms like bacteria have to cope with a multitude of antagonistic agents and environmental conditions in order to live. Under such circumstances, the bacteria may adopt a number of strategies to optimize their chances of survival [1, 5]. One such strategy is the development of genetic competence, observed in some bacterial organisms. In the competence state, specialized proteins are synthesized which allow the cell to take up large pieces of DNA from the environment and incorporate them into the bacterial genome. New traits are thus acquired from genetically distinct organisms. Experiments show that only a small fraction of the bacterial population reaches the competence state. The resulting phenotypic diversity in the population may prove to be advantageous. The individual cells in a homogeneous population share the same fate when subjected to harmful influences. Diversity enhances the chance that a fraction of the population, even if small, is able to survive and adapt to the changed circumstances. In B. subtilis, the development of competence is regulated by the transcription factor ComK synthesized by the comK gene. The protein functions as a master regulator which activates the transcription of several genes including those necessary for DNA uptake. The ComK activity in turn is controlled by a host of other proteins. An autoregulatory positive feedback module forms the core of the complex regulatory network. ComK binds to the promoter of its gene and promotes its own production. The positive feedback gives rise to bimodality in the cell population with low and high comK expression states as the stable states. In the competence state, the level of ComK proteins is high enabling ComK to act as a transcription factor. Two independent experiments [6, 7] have confirmed that an autostimulatory loop of comK expression is by itself sufficient to establish competence bimodality in a bacterial culture. The experimental findings moreover suggest that stochasticity plays an essential role in the establishment of competence.

In this paper, we study a simple model of autoregulatory positive feedback involving a single gene using both deterministic and stochastic descriptions. In the deterministic case, positive feedback and nonlinearity result in bistability in a range of parameter values. The two stable steady states correspond to low and high gene expression levels. The 
high expression state is reached when the protein level exceeds a threshold value. The autocatalytic switch is then triggered bringing about a full activation of the autostimulatory loop. In the absence of such activation the proteins are synthesized at a low level. Bimodality in a cell population requires the autocatalytic switch to be triggered in a fraction of the cell population. This is where stochasticity in gene expression comes into the picture. Several recent studies, both theoretical and experimental, highlight the significant role of stochasticity in gene expression and its regulation [8, 9, 10, 11, 12]. The two stable steady states are separated by an unstable steady state. The corresponding protein level (intermediate level of gene expression) provides the threshold for the triggering of the autocatalytic switch. The low (high) expression state is obtained when the protein level is below (above) the threshold value. Stochasticity in gene expression gives rise to fluctuations in the protein levels and the fluctuations, if sufficiently large, bring about transitions across the threshold. In the deterministic picture, bifurcations occur at two special values of the parameter $J_{0}$, the rate for basal protein synthesis. At the lower (upper) bifurcation point, there is a transition from monostability (bistability) to bistability (monostability). This framework provides an alternative explanation of population heterogenity. Inducer molecules are often required to initiate gene expression at the basal level. The distribution of the molecules may be non-uniform in a population of cells. Thus, the basal levels in the individual cells are not identical but have a disribution around an average value. If this distribution overlaps with the upper bifurcation point, the cell population develops a bimodal character. There is also a third explanation for population heterogeneity which is solely based on stochasticity in gene expression. In this case, the system is not bistable in the deterministic picture and bimodality occurs due to random transitions between the low and high expression states. In this paper, we explore the basis of bimodal protein distributions in the three scenarios outlined above. The results are interpreted in terms of the development of genetic competence in B. subtilis bacterial population.

\section{Deterministic model}

We consider a simple model of autoregulatory gene expression involving a single gene. The proteins synthesized by the gene form dimers. The dimer molecules bind to the promoter region of the gene and activate gene expression, thus constituting a positive feedback loop. Apart from autoactivation, the gene synthesizes proteins at a basal level. The detailed kinetic scheme of the model is shown in figure 1(a). The gene can be in two possible 


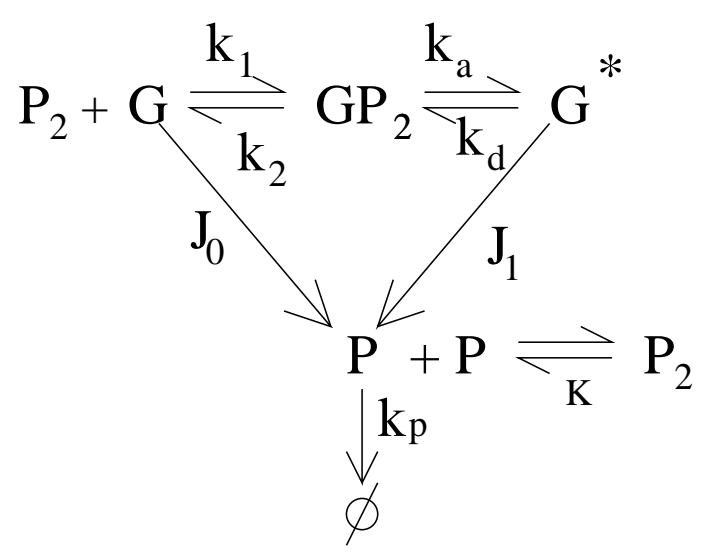

FIG. 1(a): The kinetic scheme describing autoregulatory gene expression. $G$ and $G^{*}$ are the inactive and active states of the gene. In the inactive state, proteins are synthesized at a basal rate $J_{0}$. The protein molecules form dimers $P_{2}$ which bind to the promoter region of the gene and activate the state $G$ to $G^{*}$.

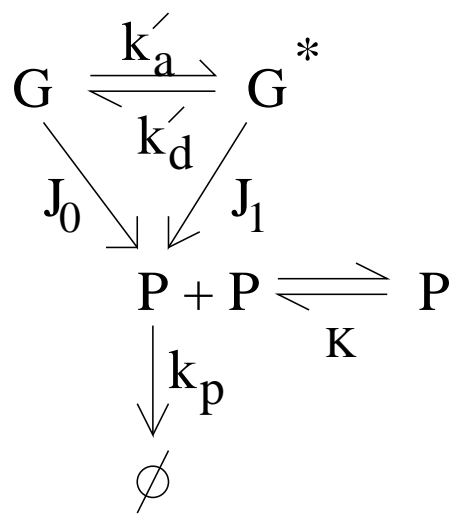

FIG. 1(b): The reduced kinetic scheme with effective activation and inactivation rate constants $k_{a}^{\prime}(x)$ and $k_{d}^{\prime}$. 
states $G$ (inactive) and $G^{*}$ (active). In the active state proteins are synthesized with rate constant $J_{1}$. In the inactive state $G$, "leaky" gene expression occurs at the basal rate $J_{0}$ $\left(J_{1}>>J_{0}\right)$. The basal rate may be enhanced using appropriate inducer molecules. The synthesized proteins dimerize with $K$ being the equilibrium dissociation constant. The protein dimer $P_{2}$ binds to the gene in its inactive state $G$ and activates the gene to the state $G^{*}$. The rate constants $k_{a}$ and $k_{d}$ are the activation and deactivation rate constants. The synthesized proteins are degraded with a rate constant $k_{p}$. The kinetic scheme in figure 1(a) can be mapped onto a simpler scheme shown in figure 1(b). The effective activation and deactivation rate constants $k_{a}^{\prime}(x)$ and $k_{d}^{\prime}$ are given by

$$
k_{a}^{\prime}(x)=k_{a} \frac{\left(x / k_{s}\right)^{2}}{1+\left(x / k_{s}\right)^{2}}, \quad \quad k_{d}^{\prime}=k_{d}
$$

where $x$ denotes the protein concentration and $k_{s}=\sqrt{\frac{k_{2}}{k_{1}} K}$.

In the simplified kinetic scheme of figure 1(b), the rate of change of protein concentration is given by

$$
\frac{d x}{d t}=\frac{J_{1} k_{a}^{\prime}(x)}{k_{a}^{\prime}(x)+k_{d}}+\frac{J_{0} k_{d}}{k_{a}^{\prime}(x)+k_{d}}-k_{p} x
$$

In the steady state, $\frac{d x}{d t}=0$ and one can identify a parameter region in which the system is bistable, i.e., has two stable steady states. These states correspond to low and high values of $x$. An unstable steady state (intermediate value of $x$ ) separates the two stable steady states. Figure 2 shows a plot of $x^{s}$ versus $J_{0}$ where $x^{s}$ denotes the steady state protein concentration. The solid branches represent stable steady states and the dotted branch, the unstable steady states. In a range of parameter $\left(J_{0}\right)$ values, the system is bistable. The other parameters have values $k_{a}=0.0008, k_{d}=0.0005, k_{s}=500.0, J_{1}=0.1$ and $k_{p}=0.0001$ in appropriate units. Bistability is, in general, accompanied by hysteresis [3, 13]. Let us assume that the system is in the lower steady state and the value of $J_{0}$ is small. As $J_{0}$ is increased (say, with the help of inducer molecules), the system continues to be in the low expression state. At a critical value $J_{0 U C}$, a discontinuous transition to the upper stable steady state occurs. If $J_{0}$ is increased further, the system is monostable, i.e., there is only one stable steady state (the upper state). If the value of $J_{0}$ is now reduced below $J_{0 U C}$, the system remains in the upper steady state which is a hallmark of hysteresis. At a lower critical value of $J_{0}=J_{0 L C}$ (marked by a vertical line on the horizontal axis of figure 2), a transition from the upper to the lower stable steady state occurs.

Hysteresis promotes robustness as once the system is in the upper stable steady state, 


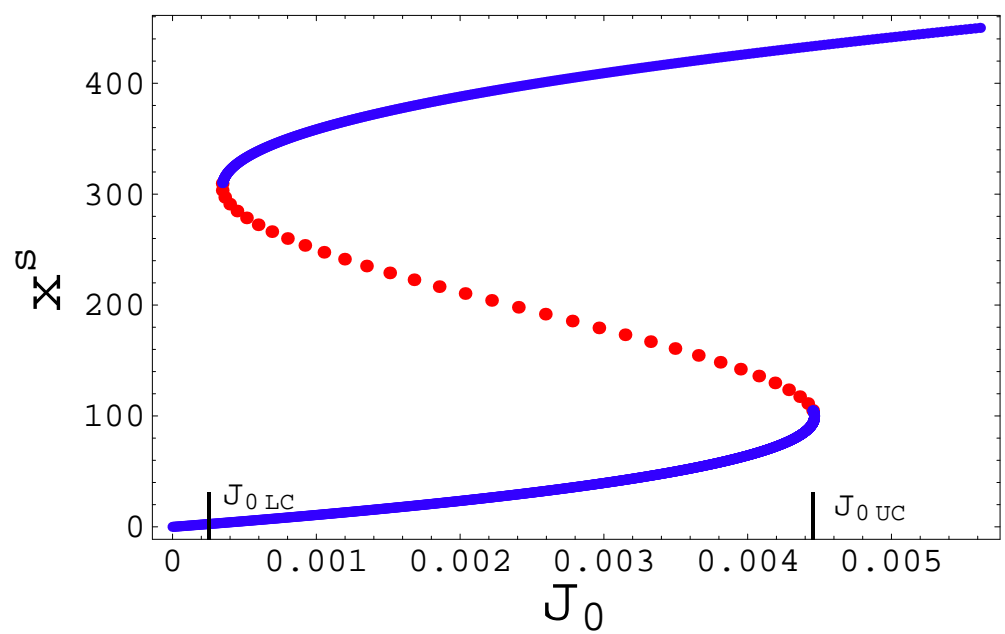

FIG. 2: Bistability and hysteresis, the solid (dotted) lines represent stable (unstable) steady states; $x^{s}$ is the steady state concentration of proteins and $J_{0}$, the basal rate of protein synthesis, serves as the bifurcation parameter. The short vertical lines on the horizontal axis denote the lower and upper bifurcation points, $J_{0 L C}$ and $J_{0 U C}$.

small fluctuations of $J_{0}$ around $J_{0 U C}$ will not give rise to a transition to the lower stable steady state. Let us now assume that the basal gene expression is initiated with the help of inducer molecules. These molecules may have a heterogeneous distribution in the cell population (each individual cell contains the autoregulatory module) which gives rise to a distribution in the basal rates $J_{0}$. If the threshold value $J_{0 U C}$ falls within the $J_{0}$ distribution, the cell population exhibits bimodality. Cells in which the basal rate $J_{0}$ is less (greater) than $J_{0 U C}$, are in the low (upper) stable steady state. Figures $3(\mathrm{a})$ and (b) illustrate this for a normal distribution of basal rates with mean $=0.00445$ and variance $=0.0005$. In figure 3(b), $p(x)$ describes the steady state distribution in the protein levels. In the steady state, $\frac{d x}{d t}=0$ in equation (2), from which the basal protein synthesis rate $J_{0}$ can be expressed as a function of $x$ i.e., $J_{0}=f(x)$. Let $p\left(J_{0}\right)$ be the distribution in basal levels $\left(J_{0} / k_{p}\right.$ is the steady state basal level). One can then write

$$
p(x)=\left.p\left(j_{0}\right)\right|_{J_{0}=f(x)}\left|\frac{d J_{0}}{d x}\right|
$$

This way of explaining bimodality is consistent with an earlier proposal on the origin of binary gene expression [14]. We now discuss the other two mechanisms for obtaining bimodality taking stochasticity in gene expression explicitly into account. 


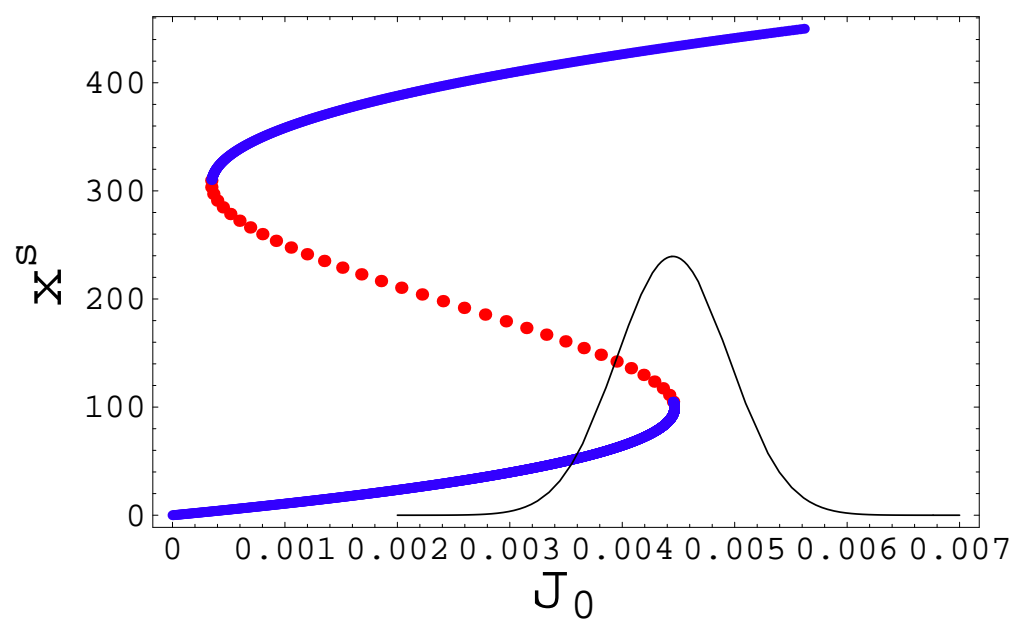

FIG. 3(a): Normal distribution describing heterogeneous inducer distribution overlaps with the upper bifurcation point $J_{0 U C}$.

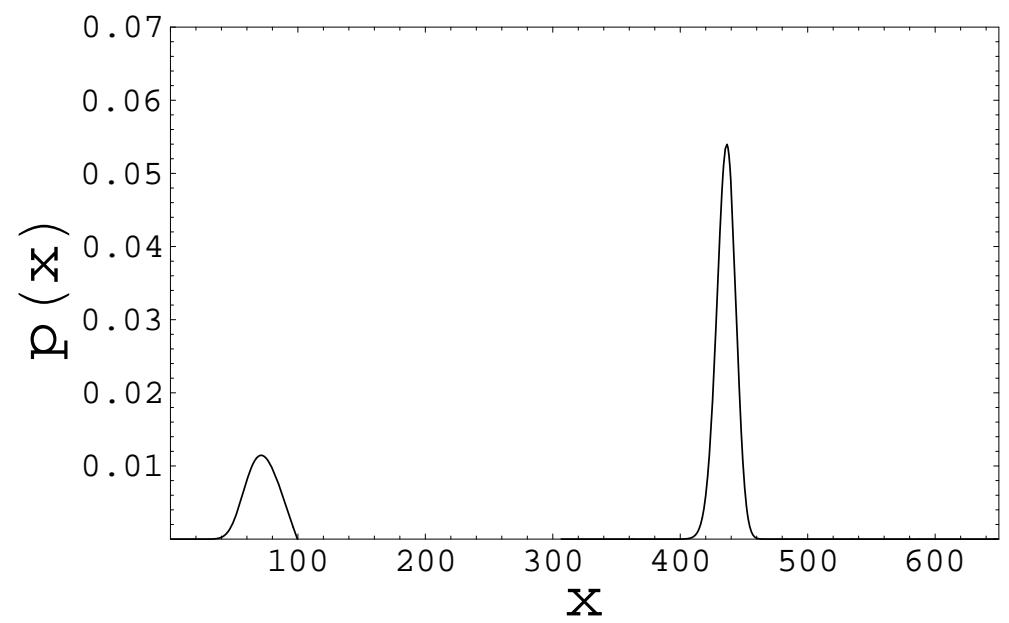

FIG. 3(b): The bimodal distribution in protein levels due to the heterogeneous distribution of inducer molecules. 


\section{Stochastic origins of bimodality}

We consider a simple stochastic model corresponding to the kinetic scheme in figure 1(b). In the model, the only stochasticity arises from the random transitions of the gene between the inactive and active states as in the minimal model of Cook et al. [15. Protein synthesis from the inactive (basal expression) and active states of the gene and protein degradation occur in a deterministic manner. We would like to determine the distribution of protein levels in the steady state of the cell population. Following the method outlined in [16], the concentration of proteins evolves as

$$
\frac{d x}{d t}=J_{1} z+J_{0}(1-z)-k_{p} x=f(x, z)
$$

where $z=1(0)$ when the gene is in the active, $G^{*}$ (inactive, $G$ ) state. The random variable $z$ switches values with stochastic rate constants $k_{a}^{\prime}(x)(0 \rightarrow 1)$ and $k_{d}^{\prime}(1 \rightarrow 0)$. Let $p_{j}(x, t)$ $(j=0,1)$ be the probability density function when $z=j$. The total probability density function is

$$
p(x, t)=p_{0}(x, t)+p_{1}(x, t)
$$

The rate of change of probability density is given by

$$
\frac{\partial p_{j}(x, t)}{\partial t}=-\frac{\partial}{\partial x}\left[f(x, j) p_{j}(x, t)\right]+\sum_{k \neq j}\left[W_{k j} p_{k}(x, t)-W_{j k} p_{j}(x, t)\right]
$$

where $W_{k j}$ is the transition rate from the state $k$ to the state $j$ and $W_{j k}$ is the same for the reverse transition. The first term in equation (6) is the so called "transport" term representing the net flow of the probability density. The second term represents the gain/loss in the probability density due to random transitions between the state $j$ and other accessible states. In the present case, equation (6) gives rise to the following two equations:

$$
\begin{aligned}
& \left.\frac{\partial p_{0}(x, t)}{\partial t}=-\frac{\partial}{\partial x}\left\{\left(J_{0}-k_{p} x\right) p(x, t)\right]\right\}+k_{d} p_{1}(x, t)-k_{a}^{\prime}(x) p_{0}(x, t) \\
& \frac{\partial p_{1}(x, t)}{\partial t}=-\frac{\partial}{\partial x}\left\{\left(J_{1}-k_{p} x\right) p_{1}(x, t)\right\}+k_{a}^{\prime}(x) p_{0}(x, t)-k_{d} p_{1}(x, t)
\end{aligned}
$$

Using equation (5), the steady state solution of equations (7) and (8) is given by

$$
p(x)=C\left(k_{p} x-J_{0}\right)^{-v}\left(J_{1}-k_{p} x\right)^{-1+\frac{k_{d}}{k_{p}}}\left(x^{2}+k_{s}^{2}\right)^{w} \operatorname{Exp}[u]
$$


where

$$
u=\frac{k_{a} \arctan \left(x / k_{s}\right) J_{0} k_{s}}{J_{0}^{2}+k_{p}^{2} k_{s}^{2}}, v=\frac{J_{0}^{2}\left(-k_{a}+k_{p}\right)+k_{p}^{3} k_{s}^{2}}{k_{p}\left(J_{0}^{2}+k_{p}^{2} k_{s}^{2}\right)}, w=\frac{k_{a} k_{p} k_{s}}{2\left(J_{0}^{2}+k_{p}^{2} k_{s}^{2}\right)}
$$

and $C$ is the normalization constant.

Figures $4(\mathrm{a})-(\mathrm{d})$ show the plots of $p(x)$ versus $x$ as the activation and deactivation rate constants $k_{a}=0.0008 * h$ and $k_{d}=0.0005 * h$ are progressively changed by varying the factor $h$. The other parameters have values $k_{s}=500, J_{0}=0.0035, J_{1}=0.1$ and $k_{p}=0.0001$. As $h$ is changed from 2 to 100, there is a transition from unimodality (figure 4(a)) to bimodality (figures 4(b) and (c)) to again unimodality (figure 4(d)). The unimodal distributions correspond to low (figure 4(a)) and high (figure 4(d)) gene expression states. In all the four cases, the deterministic dynamics (equation (2)) lead to bistability in the steady state (figure 2). The three steady state solutions for $J_{0}=0.0035$ are $x_{\text {stable } 1}=50.11, x_{\text {unstable }}=$ 160.60 and $x_{\text {stable } 2}=418.13$. The bimodality observed in figures $4(\mathrm{~b})$ and (c) are due to stochastic transitions between the stable steady states brought about by the fluctuations associated with the protein levels. The two stable steady states $x_{\text {stable } 1}$ and $x_{\text {stable } 2}$ are separated by the unstable steady state $x_{\text {unstable. When protein levels are below (above) }}$

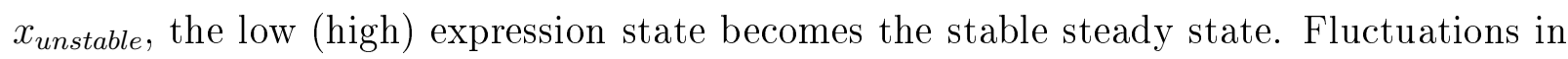
the protein levels (due to stochastic gene expression) are responsible for the excursions from one state to the other. In the deterministic picture (figure 2), the system is in the lower stable steady state for the parameter value $J_{0}=0.0035$ used in obtaining the plots in figure 4. Figures 4(b)-(d) thus clearly demonstrate that noise can alter the deterministic outcome in a significant manner. In case (d), the fluctuations associated with the lower protein level are so strong that excursions to the higher protein level occur with probability one. In terms of the autoregulatory genetic module, the autostimulatory feedback loop is fully activated when the protein level $x$ is $>x_{\text {unstable }}$ so that the high expression state is achieved in the steady state. In the deterministic picture, the time evolution of a dynamical system can be predicted with absolute certainty once the parameter values and the initial state are specified. In the present case, the different rate and binding constants constitute the parameters. The state of the system at time $t$ is given by the amount of proteins $x(t)$. The value of $x(t)$ is obtained by solving the differential equation (equation (2)) for a fixed set of parameter values and with a knowledge of the initial state $x\left(t_{0}\right)$ at time $t_{0}$. The time evolution of the system is represented by a trajectory in state space (one-dimensional in the present case). The trajectory starts from the point $x\left(t_{0}\right)$ and ends at a fixed point 
$\left(\frac{d x}{d t}=0\right)$ describing a stable steady state. In the region of bistability, the two stable steady states $x_{\text {stable } 1}$ and $x_{\text {stable } 2}$ have their individual basins of attraction [17, 18]. A trajectory which starts in one particular basin of attraction reaches the corresponding stable steady state in the course of time. The time evolution of a system stops once the steady state is reached. A steady state is stable (unstable) if the system comes back to it after a weak perturbation is applied. Small fluctuations in the protein level $x$ leave the system in the same basin of attraction. There may, however, be excursions from one basin to the other when the fluctuations are of sufficiently large magnitude. The probability of transition from one basin of attraction to the other depends amongst other factors on the value of $J_{0}$, the basal rate of protein synthesis. The gap between $x_{\text {unstable }}$ and $x_{\text {stable } 1}$ is smaller and that between $x_{\text {stable } 2}$ and $x_{\text {unstable }}$ larger as $J_{0}$ approaches $J_{0 U C}$. The reverse situation is true as $J_{0}$ approaches the lower bifurcation point. The plots in figure 4 have been obtained for progressively higher values of the activation rate constant $k_{a}$. The value of $J_{0}=0.0035$ is closer to the upper bifurcation point $J_{0 U C}$. The protein fluctuations are amplified for higher values of $k_{a}$. The fluctuations have to bridge a smaller gap for transition from the basin of attraction of $x_{\text {stable } 1}$ to that of $x_{\text {stable } 2}$ than in the case of the reverse transition. In the case of figure $4(\mathrm{a})$, the system remains in the basin of attraction of $x_{\text {stable } 1}$. As $k_{a}$ is made higher, a greater fraction of the cell population attains the high expression state. In the case of figure 4(d), almost the whole cell population is in the high expression state. Figures 5(a)-(d) show plots similar to those in figure 4 for a lower value of $J_{0}=0.0030$. The gap between $x_{\text {unstable }}$ and $x_{\text {stable } 1}$ is now larger and that between $x_{\text {stable } 2}$ and $x_{\text {unstable }}$ smaller (see figure 2). The balance in this case tilts in the favour of the lower stable steady state.

We now consider the third case in which a bimodal protein distribution has a purely stochastic origin. The system is monostable in the deterministic description. An earlier study by Kepler and Elston [19] provides examples of such cases. Some other studies have explored the basis of stochastic binary gene expression in different settings (without positive feedback) [8, 12, 20, 21]. An example in the case of autoregulated gene expression is shown in figure 6 for the parameter values $k_{a}=0.0012, k_{d}=0.0004, k_{s}=500.0, J_{0}=0.01$, $J_{1}=0.1$ and $k_{p}=0.0001$. In the deterministic description, there is only one stable steady state, $x^{s}=581.3$. The protein distribution is obtained from the analytic expression given in equation (9). The stochastic model considered in this section is analytically tractable because of certain simple assumptions. The only stochasticity considered in the model is that associated with random gene activation and deactivation. The autocatalytic feedback 

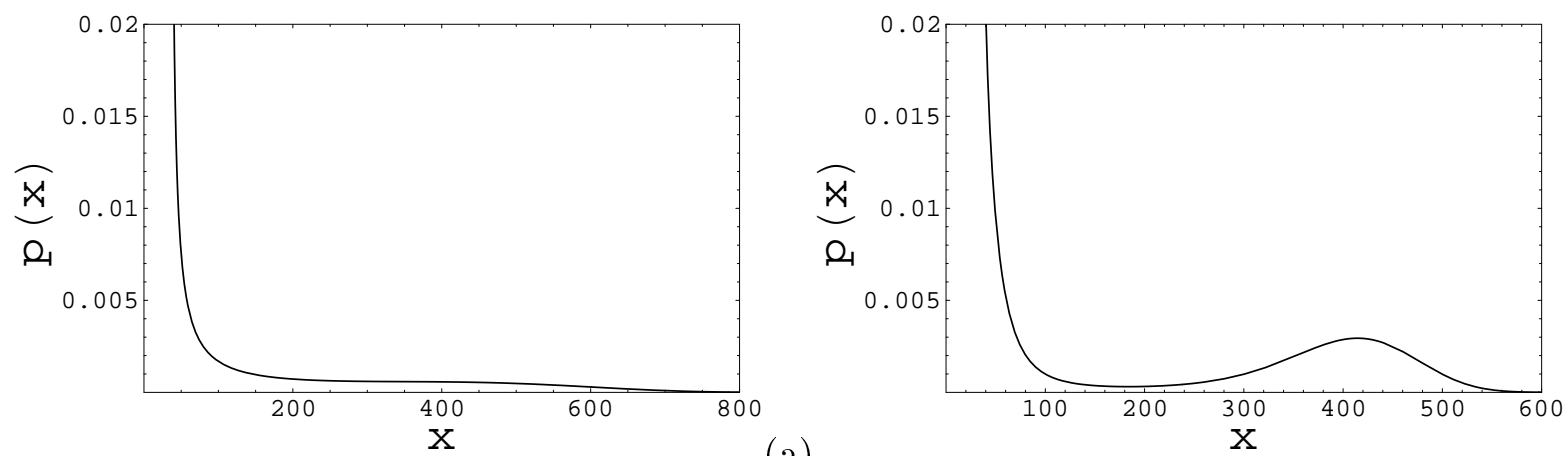

(a)
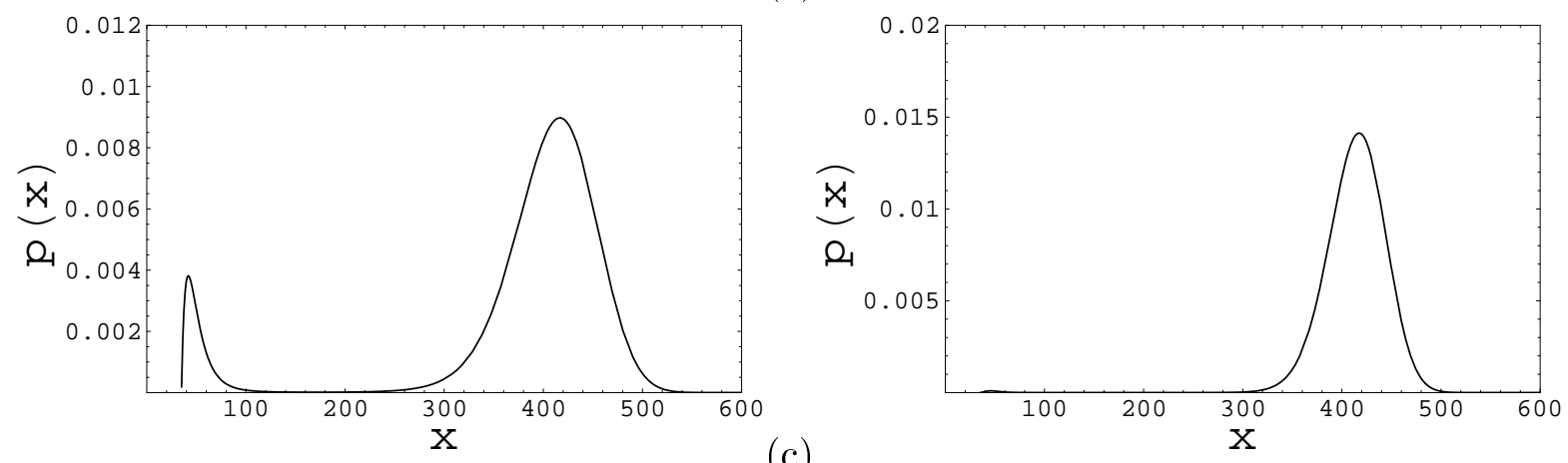

FIG. 4: The steady state distribution, $p(x)$ versus $x$, in protein levels with the activation and inactivation rate constants given by $k_{a}=0.0008 * h$ and $k_{d}=0.0005 * h$. The plots are obtained for different values of $h$, (a) $h=2$, (b) $h=20$, (c) $h=50$ and (d) $h=100$. The basal rate of protein synthesis is $J_{0}=0.0035$. The three steady states in the deterministic case are $x_{\text {stable } 1}=50.11, x_{\text {unstable }}=160.6$ and $x_{\text {stable } 2}=418.13$. 

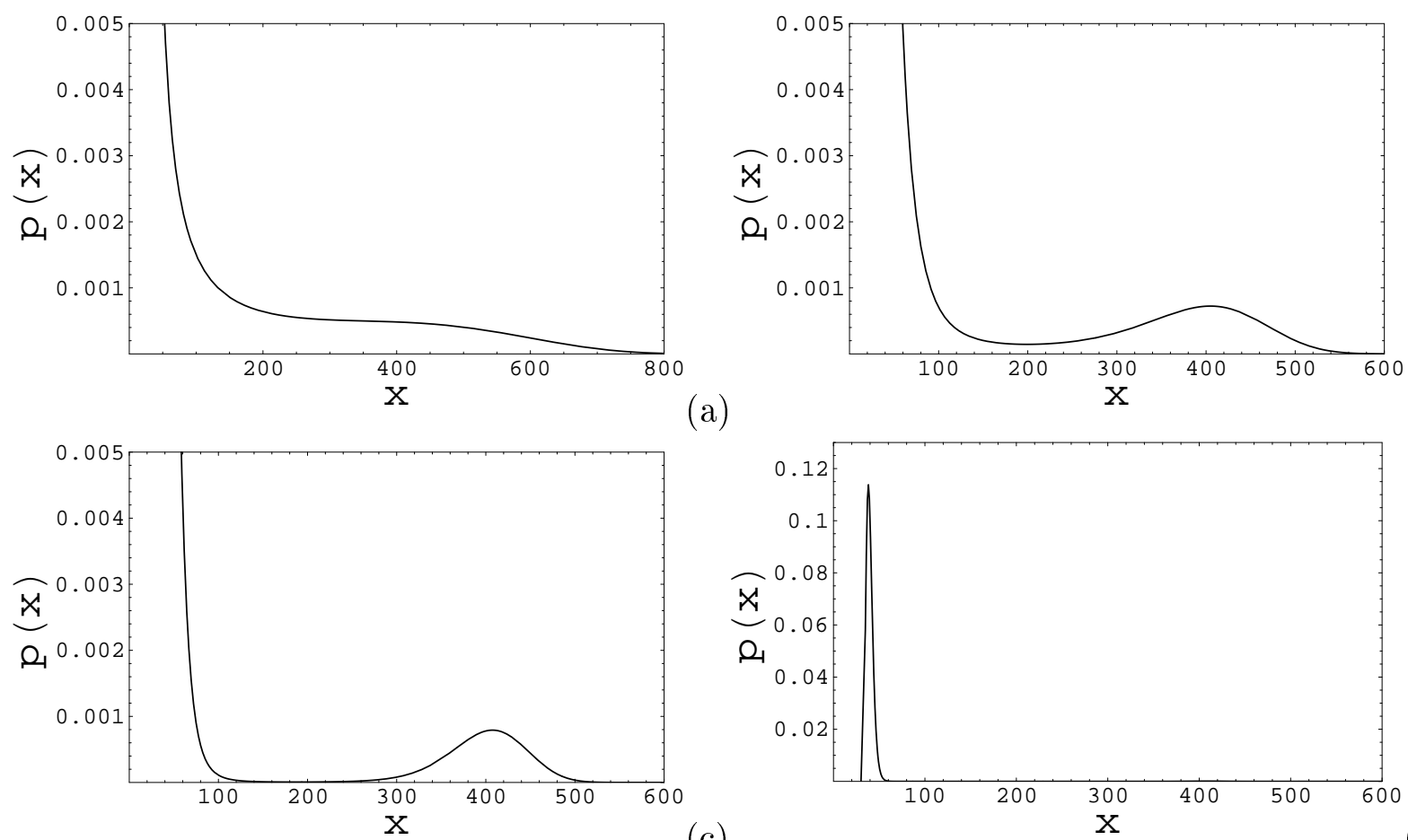

(a)

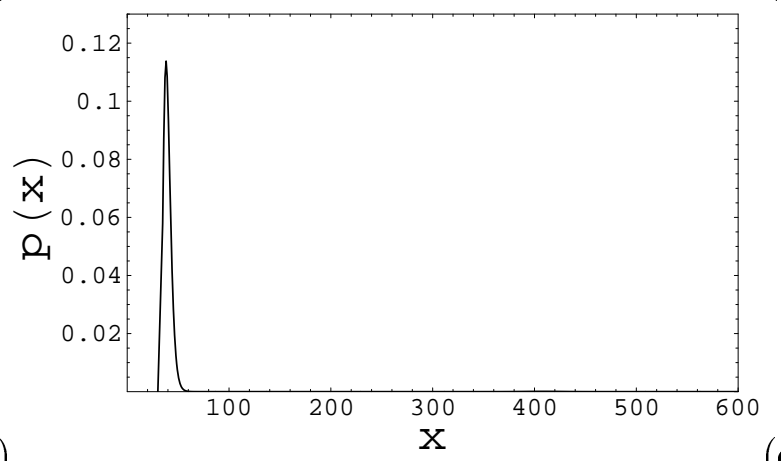

(d)

FIG. 5: The steady state distribution, $p(x)$ versus $x$, in protein levels. The parameter $J_{0}$ has the value $J_{0}=0.0030$ with $k_{a}=0.0008 * h$ and $k_{d}=0.0005 * h$ as in the case of figure 4. The values of $h$ are (a) $h=2$, (b) $h=20$, (c) $h=50$ and (d) $h=250$. The three steady states in the deterministic case are $x_{\text {stable } 1}=39.55, x_{\text {unstable }}=178.22$ and $x_{\text {stable } 2}=409.14$. 
is incorporated in an effective rate constant $k_{a}^{\prime}(x)$. The gene expression is considered as a one-step process, i.e., the intermediate stage of $m R N A$ synthesis is not explicitly taken into account. We now describe the results of a detailed simulation based on the Gillespie algorithm [22] which takes the two-step nature of gene expression into account and treats the distinct biochemical events to be stochastic in nature. The different biochemical reactions are listed in equations (11)-(21):

$$
\begin{gathered}
G+P 2 \rightarrow G P 2 \\
G P 2 \rightarrow G+P 2 \\
G P 2 \rightarrow G * \\
G * \rightarrow G P 2 \\
G * \rightarrow m \\
G \rightarrow m \\
m \rightarrow d e g . \\
m \rightarrow P \\
P \rightarrow d e g . \\
P+P \rightarrow P 2 \\
P 2 \rightarrow P+P
\end{gathered}
$$

In the above equations, the mRNA and protein are represented by $m$ and $P$ respectively, $P 2$ is a protein dimer and $G P 2$ denotes the intermediate state of a protein dimer bound to the gene in its inactive state $G$. Equations (17) and (19) describe the degradation of the mRNA and protein molecules. In the simulation, the stochastic rate constants associated with the equations (11)-(21) are $c(1)=0.003, c(2)=0.16, c(3)=0.004 * h, c(4)=0.0006 * h$, $c(5)=0.1, c(6)=0.0015, c(7)=0.0001, c(8)=0.000001, c(9)=0.008, c(10)=0.01$ and $c(11)=0.01 \mathrm{in}$ appropriate units. The simulation is carried out for three different values of $h=1,10$ and 200 respectively. The results are shown in figures $7(\mathrm{a})$-(c). The plots on the left show the time trajectories, $x(t)$ versus $t$, where $x(t)$ is the amount of proteins at time $t$. The plots on the right show the distributions $p(x)$ versus $x$ on repeating the simulation 3000 times. The quantity $p(x) d x$ provides a measure of the fraction of cells in a 


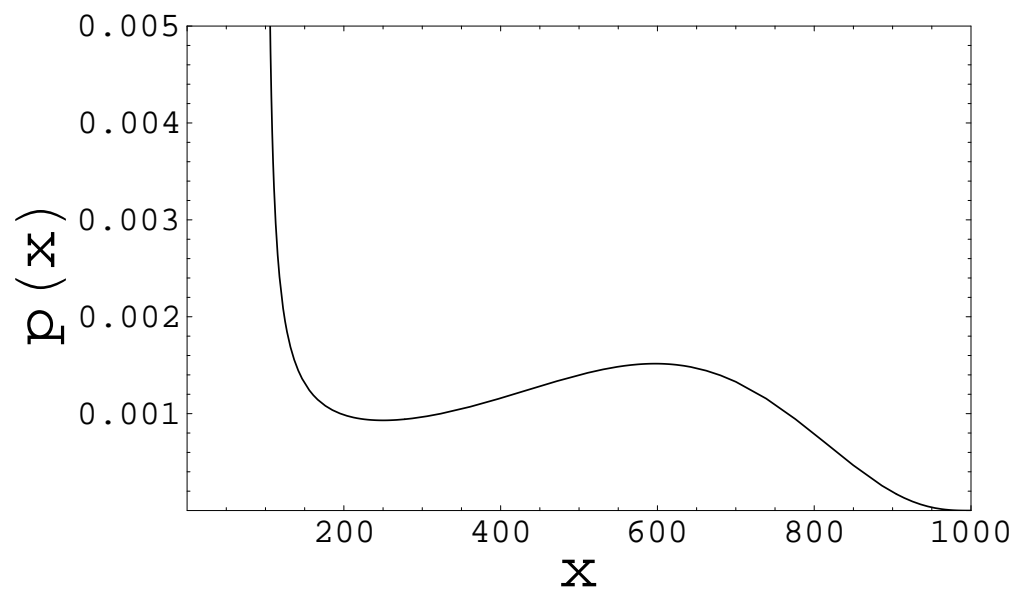

FIG. 6: The steady state distribution, $p(x)$ versus $x$, in protein levels for $k_{a}=0.0008$, $k_{d}=0.0004$ and $J_{0}=0.001$. The bimodal distribution has a purely stochastic origin. In the deterministic case, there is only one stable steady state $x^{s}=581.3$.

population with protein levels between $x$ and $x+d x$. For the parameter values used in the simulation, only a small fraction of cells is in the high expression state. Figures 7(a) and 7(b) show that the stochastic nature of the biochemical events involved in gene expression is responsible for a bimodal protein distribution.

\section{Discussions}

In this paper, we study how positive feedback combined with stochasticity gives rise to binary gene expression, i.e., a bimodal distribution in the protein levels in a population of cells. There are some earlier studies [2, 19] on the same issue but the modeling detail and context are different. The motivation for the present study comes from the experimental observation that a single comK gene, which autoregulates its expresssion via a positive feedback loop, is by itself sufficient to generate heterogeneity in a population of B. subtilis [5, 6, 7]. A fraction of the cell population develops competence due to the high expression state of comK. This is so when the ComK protein level exceeds a threshold value thus triggering the full activation of the autostimulatory loop. The comK autoregulatory genetic module is at the core of a complex network of molecular interactions which regulate comK transcription and the stability of the ComK proteins. In this case, only a small fraction of cells, about ten percent, develops competence. When experiments are carried out on the isolated genetic module, the fraction of cell population in the high comK expression 


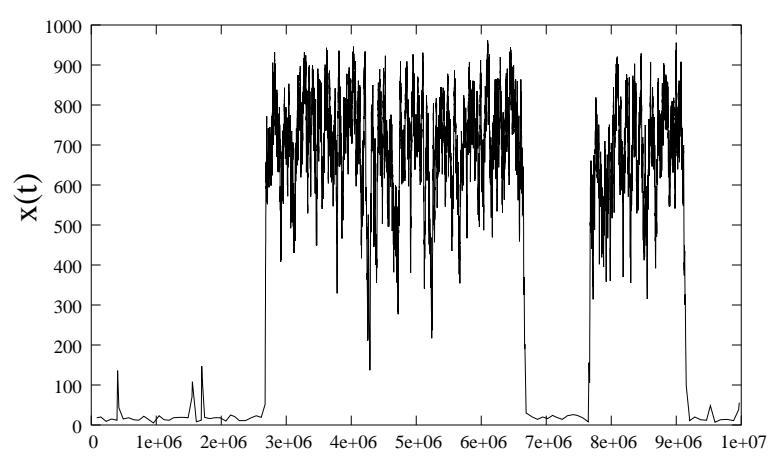

Time (t)

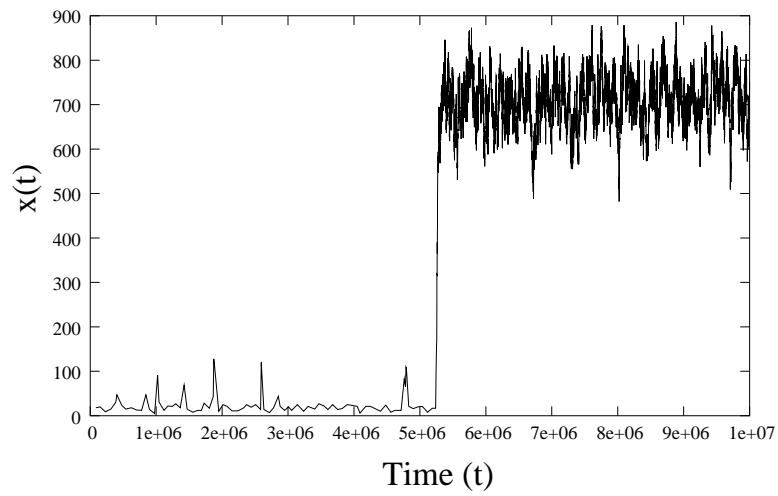

Time (t)

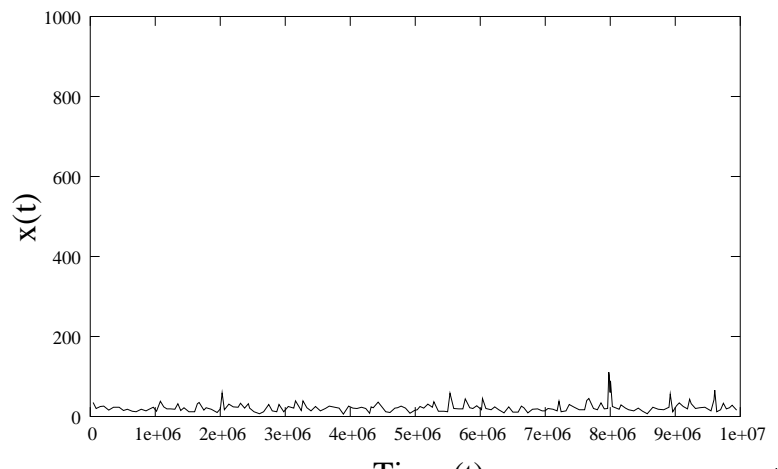

Time (t) $1(\mathrm{a})$

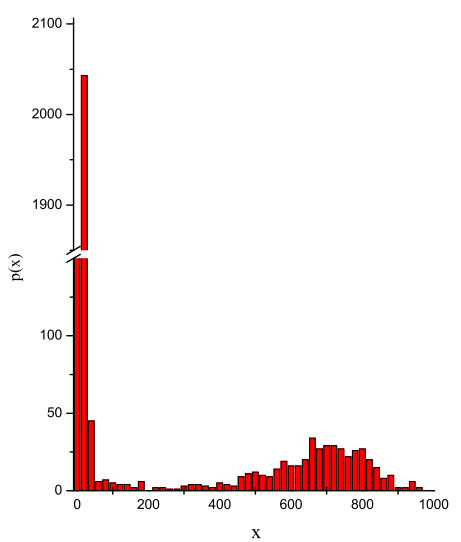

$2(\mathrm{a})$

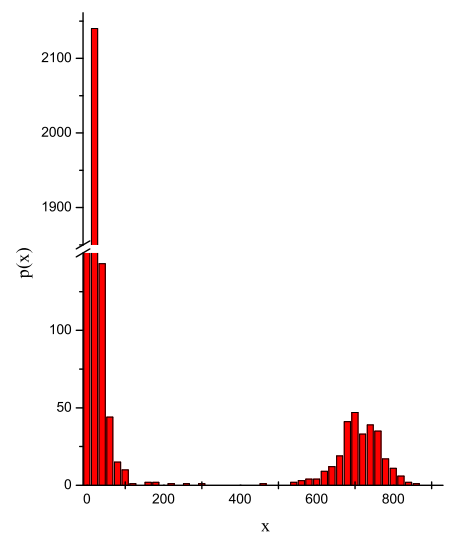

$1(\mathrm{~b})$

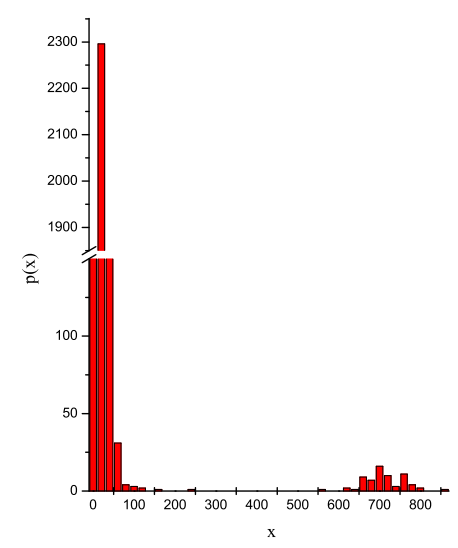

$1(\mathrm{c})$

FIG. 7: Results of simulation based on the Gillespie algorithm. The stochastic rate constants for gene activation and inactivation are $c(3)=0.004 * h$ and $c(4)=0.0006 * h$ The factor $h$ has values (a) $h=1$, (b) $h=10$ and (c) $h=200$. The plots in the first column show the variation of protein amount as a function of time. The plots in the second column show the corresponding steady state distributions, $p(x)$ versus $x$. 
state can be quite large. It has further been suggested that stochasticity in gene expression is responsible for throwing the autocatalytic switch [5, 6, 7]. In our study, we focus only on the core module, namely, the comK autoregulatory genetic module of the network regulating competence development. The module represents a single gene (comK), the protein product of which autoactivates its own synthesis through dimerization and subsequent binding at the appropriate region of the DNA. Our model incorporates these minimalist features of the autoregulatory comK module. We have explored the basis of binary gene expression in the framework of three different possibilities. In the first two cases, the underlying dynamics lead to bistability in a deterministic description. A heterogeneous distribution in inducer molecules may give rise to a bimodal distribution in the protein levels. In the second and third cases, we take stochasticity in gene expression into account and derive an analytic expression (equation (9)) for the steady state distribution of protein levels. The analytical tractability of the stochastic model arises from two assumptions. Firstly, the two major steps of gene expression, namely, transcription (synthesis of mRNAs) and translation (synthesis of proteins) are combined into a single step leading to protein production. Secondly, the only source of stochasticity in the model lies in the random activation and deactivation of the target gene expression. The first assumption provides the basis for several studies of stochastic gene expression [15, 19, 21, 23]. The second assumption is strictly valid when the dominant source of noise is associated with the random activation and deactivation of gene expression. This is so in the case of slow promoter kinetics. As discussed in detail in [12], slow transitions between the promoter states result in transcriptional bursts of mRNA synthesis and increased heterogeneity within a cell population including bimodal protein distributions. Experimental evidence of transcriptional bursting has been obtained for both prokaryotes and eukaryotes [24, 25]. A recent experiment on stochastic mRNA synthesis in mammalian cells [26] shows that the mRNA levels display large cell-to-cell variations due to random, infrequent activation of gene expression. The statistics of the variations are adequately described by a model in which the only source of stochasticity lies in the random activation and deactivation of the gene. There could be a number of factors which lead to slow transitions between the promoter states. Chromatin remodeling has been conjectured to cause transcriptional bursts in eukaryotic systems [12]. In both prokaryotes and eukaryotes, pulsatile gene expression may also result from regulatory molecules binding at and unbinding from the DNA sites, DNA undergoing conformational changes so that the RNA polymerase has only brief access to the promoter region etc. In the case of $E$. coli, there is experimental evidences 
that long periods of inactivity are interspersed by shorter periods when the gene is in the active state [24]. An earlier result of Ozbudak et al. on B. subtilis [8] has been reanalyzed to show that the data are not inconsistent with the possibility of transcriptional bursts (random gene activation-inactivation) [24]. Our simple stochastic model, based on random transitions between inactive and active gene states, is thus consistent with experimental reality. In the case of bistability, stochasticity triggers transitions between the two stable steady states which is responsible for bimodal protein distributions. In the case when the system is monostable in a deterministic description, binary gene expression can still occur due to a combination of positive feedback and stochastic transitions between the inactive and active states of the gene. Our analytical results are supported by the simulation results in the case of a more detailed stochastic model in which transcription and translation are treated as separate processes and stochasticity associated with all the biochemical steps (equations (11)-(21)) are taken into account. In both the cases, the results are valid over a wide range of parameter values. We now briefly discuss the experimental possibility for distinguishing between the three mechanisms discussed in the paper. As shown in figure 2, bistability implies hysteresis. A properly designed experiment can detect hysteresis in the response $\left(x^{s}\right.$ in figure 2$)$ as the variable along the $x$-axis ( $J_{0}$, the basal rate of protein synthesis in figure 2) is changed. Discontinuous jumps in response at the bifurcation points and a non-reversible response are the hallmarks of hysteresis. The value of $J_{0}$ may be changed using appropriate inducer molecules. One can use a cell sorter and separate a subpopulation from a bimodal cell population. The subpopulation develops bimodality in the course of time if there are stochastic transitions between the low and high expression levels.

Süel et al. [27 have investigated competence development on the basis of a model describing an excitable stochastic system. The key ingredients of the model are: the comK autoregulatory loop, the inhibition of ComK degradation by ComS proteins and repression of the comS gene by ComK. Theoretical analysis of the model dynamics is combined with experiments to gain insight on the entry into and exit from the competence state. This state corresponds to an unstable fixed point of the model dynamics. The system has only one stable steady state in which the ComK level is low. Fluctuations in the levels of ComK/ComS excite the system into the competence state with eventual return to the noncompetence state. In the excitable system, repeated stochastic triggering of the competence state is thus possible. Some of the premises of the model like the "indirect" repression of the comS gene by ComK need experimental confirmation under 
wild-type expression conditions [28]. The study nonetheless is an elegant example of how model studies combined with experiments can provide a new perspective on noise-induced phenomena in biological systems. Our model has the comK autoregulatory loop as the sole ingredient and focuses on the specific experiment by Smits et al. [1] on the single autoregulatory module. Genetic competence in B. subtilis provides a concrete example of a natural system in which a single gene, regulating its expression via an autoregulatory positive feedback loop, is by itself sufficient to establish two types of stable states in the cell population. Recently, two groups have independently discovered a similar phenomenon in the human fungal pathogen Candida albicans [29, 30]. In both the cases, the autoregulatory modules are parts of complex genetic circuitry. The single gene modules almost exclusively control the cellular switch operating between two stable states. The resulting heterogeneity is epigenetic in nature. B. subtilis and Candida albicans thus illustrate the essentiality and sufficiency of network modules in explaining particular types of biological function. The role of the other components of the associated regulatory networks lies in modulating the functional response. In B. subtilis, several genes regulate the expression of the comK gene the protein product of which regulates the expression of several other genes. The products of the regulatory genes modulate the threshold for the triggering of the autocatalytic switch and influence the stability of the ComK proteins. The additional circuitry probably includes features which further stabilize the steady states. In Candida albicans, the WOR1 gene acts as the master regulator. The gene autoregulates its own expression via a positive feedback loop. The switch now operates between the cellular states: white and opaque. The two types of cells, white and opaque, differ in their morphologies, the genes they express, the host tissues in which they are resident and also in their mating characteristics. In the white cells, WOR1 is expressed at low levels whereas the levels are high in the opaque state. As in the case of B. subtilis, stochasticity appears to drive the transitions between the two types of cell. The results derived in this paper, specially those pertaining to the combined effects of bistability and stochasticity, should be of relevance in explaining the white-opaque switching in Candida albicans.

\section{Acknowledgement}

I. B. thanks W. K. Smits for some helpful discussions. R.K. is supported by the Council of Scientific and Industrial Research, India under Sanction No. 9/15 (239)/2002-EMR-1. 


\section{References}

[1] Smits W K, Kuipers O P and Veening J-W 2006 Phenotypic variation in bacteria: the role of feedback regulation Nat. Rev. Microbiol. 4 259-271.

[2] Isaacs F J, Hasty J, Cantor C R and Collins J J 2003 Prediction and measurement of an autoregulatory genetic module Proc. Natl. Acad. Sci. 100 7714-7719.

[3] Ferrell J E Jr 2002 Self-perpetuating states in signal transduction: positive feedback, double negative feedback and bistability Curr. Opin. Cell Biol. 14 140-148.

[4] Becskei A, Seraphin B and Serrano L 2001 Positive feedback in eukaryotic gene networks: cell differentiation by graded to binary response conversion EMBO J 202528 2535 .

[5] Dubnau D and Losick R 2006 Bistability in Bacteria Mol. Microbiol. 61 564-572.

[6] Smits W K et al. 2005 Stripping Bacillus: ComK auto-stimulation is responsible for the bistable response in competence development Mol. Microbiol. 56 604-614

[7] Maamar H and Dubnau D 2005 Bistability in the Bacillus subtillis K-state (competence) system requires a positive feedback loop Mol. Microbiol. 56 615-624.

[8] Ozbudak E M, Thattai M, Kurtser I, Grossman A D and van Oudenaarden A 2002 Regulation of noise in the expression of a single gene Nature Genet. 31 69-73.

[9] Swain P S, Elowitz M B and Siggia E D 2002 Intrinsic and extrinsic contributions to stochasticity in gene expression Proc. Natl. Acad. Sci. U. S. A. 99 12795-12800.

[10] Blake W J, Kaern M, Cantor C R and Collins J J 2003 Noise in eukaryotic gene expression Nature 422 633-37; Blake et al. 2006 Phenotypic consequences of promotermediated transcriptional noise Moleculer Cell 24 853-865.

[11] Raser J M and O'Shea E K 2005 Noise in gene expression: origins, consequences and control Science 309 2010-2013 and referrences therein.

[12] Kaern M, Elston T C, Blake W J and Collins J J 2005 Stochasticity in gene expression: From theories to phenotypes Nat. Rev. Gen. 6, 451-464 and references therein. 
[13] Tyson J J, Chen K C and Novak B 2003 Sniffers, buzzers, toggles and blinkers: dynamics of regulatory and signaling pathways in the cell Curr. Opin. Cell Biol. 15 221-231.

[14] Louis M and Becskei A 2002 Binary and graded responses in gene networks Sci. STKE (doi:10.1126/stke.2002.143.pe33).

[15] Cook D L, Gerber A N and Tapscott S J 1998 Stochastic gene expression: Implications for haploinsufficiency Proc. Natl. Acad. Sci. 95 15641-15647.

[16] Karmakar R and Bose I 2004 Graded and binary responses in stochastic gene expression Phys. Biol. 1 1-8.

[17] Strogatz S H 1994 Nonlinear dynamics and chaos: with applications to physics, biology, chemistry and engineering (Cambridge: Perseus Books).

[18] Toulouse T, Ao P, Shmulevich I and Kauffman S 2005 Noise in a small genetic circuit that undergoes bifurcation Complexity 11 45-51.

[19] Kepler T B and Elston T C 2001 Stochasticity in transcriptional regulation: origins, consequences and mathematical representations. Biophys. J. 81 3116-3136.

[20] Ko M S H 1991 A stochastic model for gene expression J. Theor. Biol. 153 181-194.

[21] Pirone J R and Elston T C 2004 Fluctuations in transcription factor binding can explain the graded and binary responses observed in inducible gene expression $J$. Theor. Biol. 226 111-121.

[22] Gillespie D T 1977 Exact stochastic simulation of coupled chemical reactions J. Phys. Chem. 81 2340-2361.

[23] Peccoud J and Ycart B 1995 Markovian modeling of gene expression Theor. Pop. Biol. 48 222-234.

[24] Golding I, Paulsson J, Zawilski S M and Cox E C 2005 Real-time kinetics of gene activity in individual bacteria Cell 123 1025-1036.

[25] Long C, Friedman N and Xie X S 2006 Stochastic protein expression in individial cells at the single molecule level Nature 440 358-362. 
[26] Raj A, Peskin C S, Tranchina D, Vargas D Y and Tyagi S 2006 Stochastic mRNA Synthesis in Mammalian cells PLoS Biology 4 1707-1719.

[27] Süel G, Garcia-Ojalvo J, Liberman L and Elowitz M B 2006 An excitable gene regulatory circuit induces transient cellular differentiation Nature $\mathbf{4 4 0}$ 545-550.

[28] Samoilov M S, Price G and Arkin A P 2006 From fluctuations to phenotypes: the physiology of noise Sci. STKE (doi: 10.1126/stke.366 2006 re17)

[29] Zordan R E, Galgoczy D J and Johnson A D 2006 Epigenetic properties of whiteopaque switching in Candida albicans are based on a self-sustaining transcriptional feedback loop Proc. Natl. Acad. Sci. 103 12807-12812.

[30] Huang G, Wang H, Chou S, Nie X, Chen J and Liu H 2006 Bistable expression of WOR1, a master regulator of white-opaque switching in Candida albicans Proc. Natl. Acad. Sci. 103 12813-12818. 